\title{
Left-sided pancreatic incidentalomas treated with laparoscopic approach: a report of 20 cases
}

\author{
Marco Chiarelli', Martino Gerosa ${ }^{4}$, Fulvio Tagliabue ${ }^{1}$, Luca Fumagalli' ${ }^{1}$ Angelo Guttadauro ${ }^{2}$, Francesco Gabrielli ${ }^{2}$, \\ Alessandro Marando ${ }^{3}$, Matilde De Simone ${ }^{5}$ and Ugo Cioffi ${ }^{5 *}$
}

\begin{abstract}
Background: The diffusion of cross-sectional imaging has recently permitted the detection of an increasing number of incidentalomas localized in the distal pancreas.

Currently, there are no studies in the literature exploring the laparoscopic approach as treatment for left-sided pancreatic incidentalomas.

Methods and results: We report a series of 20 incidentalomas localized in the body and tail of the pancreas treated with laparoscopic surgery over the period 2010-2014. The incidental masses of our series included a great variety of histotypes and a relevant proportion of malignant lesions. In two cases, the laparoscopic procedures were converted to open surgery. No postoperative death was observed. The postoperative pancreatic fistula rate was $20 \%$, and the new-onset diabetes rate was $25 \%$.

Conclusions: Left-sided pancreatic incidentalomas in patients with minor comorbidities can be safely treated with laparoscopic approach. Only clinical trials will confirm whether laparoscopic surgery is an effective treatment for malignant lesions.
\end{abstract}

Keywords: Pancreatic incidentaloma, Distal pancreatectomy, Pancreas, Laparoscopic surgery

\section{Background}

Pancreatic incidentalomas (PIs) are asymptomatic masses accidentally diagnosed by radiological, endoscopic, or laboratory exams performed for symptoms not suggesting pancreatic diseases [1]. Masses confined to the body and tail of the gland are frequently asymptomatic, but recently, an increasing number of these lesions has been detected, due to the large use of cross-sectional imaging [2]. To date, only few series of PIs have been reported in the literature, and consequently, some aspects of their management are still debated [3-7].

Laparoscopic distal pancreatectomy (LDP) is actually considered an effective and safe treatment for benign and premalignant left-sided pancreatic tumors [8-11]. Minimally invasive surgery could be a good choice for the treatment of incidental masses of the distal pancreas,

\footnotetext{
* Correspondence: ugo.cioffi@guest.unimi.it

${ }^{5}$ Department of Surgery, University of Milan, Milan, Italy

Full list of author information is available at the end of the article
}

but currently, there are no studies in the literature confirming this hypothesis.

We report a series of 20 incidentalomas localized in the body and tail of the pancreas treated with laparoscopic approach.

\section{Methods \\ After obtaining local ethics committee approval, we retrospectively reviewed the medical records of all the patients who underwent distal pancreatectomy at the General Surgery Division of Alessandro Manzoni Hospital, from January 2010 to December 2015. Our institution is categorized as a medium-volume hospital for pancreatic surgery [12]. Only patients with asymp- tomatic incidentally identified lesions treated with laparoscopic approach were included into the study. The data collected were as follows: preoperative data-age, gender, ethnicity, body mass index (BMI), American Society of Anesthesiologists (ASA) physical status}


classification, indication and type of imaging exams, and tumor size and location; intraoperative data-type of resection and operative time; pathological diagnosis and staging (according to the 7th edition of American Joint Committee on Cancer TNM staging system); and postoperative outcomes-perioperative mortality, length of hospital stay, readmission, postoperative pancreatic fistulas (POPF) [13], post-pancreatectomy diabetes (PPD) [14], and generic complications.

LDP was performed according to the standard technique described in the literature [15]. The pancreas was transected with a linear cutting stapler (Endopath ${ }^{\circ}$ ETS Linear Cutter-Ethicon Endo-Surgery Inc., Cincinnati $\mathrm{OH}, \mathrm{USA}$ ); no extra suture was performed routinely. One suction drain was left close to the transected pancreas.

In patients with malignant neoplasm, the radiological follow-up consisted in CT scan every 6 months. Informed consent for publishing personal data was obtained from each patient included in the study.

\section{Results}

We retrospectively collected 34 cases of tumors of the distal pancreas that underwent surgery during the period 2010-2015: 22 patients (64.7\%) were asymptomatic and $20(58.8 \%)$ were treated with laparoscopic approach. The mean age was 63.4 years (range 26-78). Fourteen patients were female and 6 male. All patients were Caucasian. The median BMI was 24.75-interquartile (IQ) range 23.726.2. Four patients were classified ASA 1 (20\%), 12 patients ASA 2 (60 \%), and 4 patients ASA $3(20 \%)$. The main radiological, pathological, and surgical characteristics of the series are summarized in Tables 1 and 2.

In 12 patients, diagnosis was made during diagnostic work-up in the emergency department (ED). In three patients, the suspected diagnosis was pulmonary embolism; in two, colonic diverticulitis; and in one, pericarditis. In three patients presenting with hematuria and three with renal stones, PI was an incidental finding.

In six cases, imaging was performed for follow-up (two for pelvic cyst, two for uterine carcinoma, and one for prostate carcinoma) or for staging (one for oral neoplasm). In two patients, PI was detected due to an increase of serum amylases.

Radiological diagnosis was made by abdominal ultrasonography (US) in 5 patients and computed tomography (CT) scan in 15 patients. In nine cases, CT was performed in ED.

In two cases, the laparoscopic procedures were converted to open surgery for the large size of PI. The median operative time was $203.5 \mathrm{~min}$ (IQ range 193-218.5). The median postoperative hospital stay was 7.5 days (IQ range 6-10). Four patients (20\%) developed POPF: 2 were grade $A$ and 2 grade $B$ fistulas. No emergent reintervention was required and conservative management was adopted in all cases. Two patients with grade B fistulas were treated with enteral nutrition and antibiotic therapy; the drainage was maintained in place until leakage resolution. In one case, the patient was discharged with drain in situ and reevaluated in an outpatient setting. In our series, the median persistency of POPF was 16 days. In 4 cases (20\%), postoperative pleural effusion was observed: in 2 patients, it was associated with grade B pancreatic fistulas, and in 2 cases, it was secondary to pneumonia; one patient required thoracentesis. Five patients $(25 \%)$ developed PPD during the postoperative course. In our series, no case of postoperative death or readmission was observed at 90day follow-up.

In 8 patients (40\%), the PI was located in the body of the gland; in 9 patients (45\%), in the tail; and in 3 patients (15\%), between the body and tail.

In all the patients, the resection margins were negative for tumor involvement. Histology revealed ductal adenocarcinoma (DAC) in six patients-associated with undifferentiated carcinoma and intraductal papillary mucinous neoplasm (IPMN) in two patients; neuroendocrine tumor in five patients (two presented the cystic variant); and two cases of acinar cell carcinoma (ACC). Serous cystic neoplasm was detected in two patients, mucinous cystic neoplasm in two patients, solid pseudopapillary neoplasm in two patients, and one patient showed an isolated IPMN.

The mean follow-up of the cohort was 31 months. All the patients with cystic neoplasms or neuroendocrine tumors were alive without disease recurrence in December 2015. In 8 patients with DAC and ACC, the median number of lymph nodes removed was 15 (IQ range 13.5-17). In this specific sub-group, pathological staging was stage I $\mathrm{B}$ in one patient, stage II A in four patients, and stage II B in three patients. The median follow-up time of this subgroup was 17 months: six of eight patients died of tumor recurrence, while two patients are alive in December 2015 (one with disease recurrence).

\section{Discussion}

The preoperative work-up of a pancreatic lesion should determine its nature in order to plan the most accurate treatment. Nevertheless, in some cases PIs are not easy to characterize preoperatively [2]. Incidental masses of the pancreas include a great variety of lesions and unusual histotypes are frequently counted in the series present in the literature $[4,5,7]$. Differently from DAC, uncommon histological types with a lower biological aggressiveness, such as mucinous cystic neoplasms or neuroendocrine tumors, are preferentially located in the distal pancreas [16-18]: in our series, we found two ACC, two cystic neuroendocrine tumors, and two solid pseudopapillary neoplasms (Figs. 1, 2, and 3). Furthermore, in about $7 \%$ of patients with a pancreatic mass is 
Table 1 Demographic, radiological, and pathological data of 20 cases of left-sided pancreatic incidentalomas treated with laparoscopic approach

\begin{tabular}{|c|c|c|c|c|c|c|c|c|}
\hline No. & Age & Sex & BMl & ASA score & Imaging & Location & Size $(\mathrm{cm})$ & Histology \\
\hline 1 & 69 & $\mathrm{~F}$ & 21.4 & 2 & CT-MRI & Body & 4.5 & $\mathrm{SCN}$ \\
\hline 2 & 39 & F & 22.9 & 1 & US-CT & Body-tail & 9.0 & SPPN \\
\hline 3 & 62 & M & 25.7 & 1 & $C T$ & Tail & 1.5 & NET \\
\hline 4 & 65 & M & 23.7 & 2 & CT-MRI & Body & 3.5 & IPMN \\
\hline 5 & 55 & F & 23.9 & 2 & CT-MRI & Body-tail & 4.5 & $\mathrm{MCN}$ \\
\hline 6 & 75 & F & 26.1 & 3 & CT & Tail & 3.8 & ACC \\
\hline 7 & 72 & M & 32.6 & 2 & CT & Body & 5.4 & $D A C+U C$ \\
\hline 8 & 72 & F & 23.7 & 2 & US-CT & Body & 4.5 & DAC \\
\hline 9 & 71 & F & 25.8 & 2 & CT-EUS & Body & 3.7 & ACC \\
\hline 10 & 61 & F & 24.8 & 2 & CT-MRI & Tail & 3.5 & $\mathrm{SCN}$ \\
\hline 11 & 26 & F & 24.2 & 1 & US-CT-MRI & Tail & 9.5 & SPPN \\
\hline 12 & 72 & F & 31.4 & 3 & CT-MRI & Tail & 2.4 & IPMN + DAC \\
\hline 13 & 76 & $\mathrm{~F}$ & 24.7 & 2 & CT-EUS & Body & 1.6 & DAC \\
\hline 14 & 49 & $\mathrm{~F}$ & 22.3 & 1 & CT-EUS & Tail & 2.5 & NET \\
\hline 15 & 78 & $F$ & 27.3 & 3 & US-CT-MRI & Body-tail & 6.2 & $\mathrm{MCN}$ \\
\hline 16 & 52 & M & 27.8 & 2 & $C T$ & Tail & 5.3 & CNET \\
\hline 17 & 75 & M & 24.7 & 3 & CT-EUS & Body & 2.5 & DAC \\
\hline 18 & 67 & F & 26.3 & 2 & US-CT & Tail & 1.8 & NET \\
\hline 19 & 65 & M & 24.8 & 2 & CT-EUS & Tail & 3.0 & DAC \\
\hline 20 & 67 & $\mathrm{~F}$ & 21.5 & 2 & CT-MRI & Body & 4.5 & CNET \\
\hline
\end{tabular}

$B M I$ body mass index, ASA American Society of Anesthesiologists, US ultrasonography, CT computed tomography, MRI magnetic resonance imaging, EUS endoscopic ultrasound, $S C N$ serous cystic neoplasm, $M C N$ mucinous cystic neoplasm, SPPN solid pseudopapillary neoplasm, IPMN intraductal papillary mucinous neoplasm, NET neuroendocrine tumor, CNET cystic neuroendocrine tumor, ACC acinar cell carcinoma, DAC ductal adenocarcinoma, UC undifferentiated carcinoma LDP

not possible to establish a definitive diagnosis before surgery despite a complete preoperative imaging [19].

The diagnostic work-up of our series was characterized by the exiguity of preoperative exams. Solid masses localized in the distal pancreas are very frequently suitable for surgical resection, and consequently, CT findings are sufficient to plan the more appropriate management of a left-sided incidentaloma in the majority of the cases [7, 20].

All patients underwent a contrast-enhanced multidetector CT scan with a biphasic protocol (arterial and venous phases). Currently, intravenous contrastenhanced CT scan is considered the pivotal radiological technique for the detection, definition, and staging of pancreatic masses [21-23]. In the last decade, the use of CT imaging increased by threefold in emergencytreated patients [24]: in our series, eight cases of PI were discovered by a CT scan performed in patients evaluated in the ED.

A relevant proportion of PIs were detected by transabdominal US. US is considered the first-level imaging in hepato-biliary disease, but the deep location of the pancreas and the operator dependency make the US an exam with low accuracy for the correct assessment of a pancreatic tumor [21].

Magnetic resonance imaging (MRI) and endoscopic ultrasound (EUS) were performed in selected cases. A complete pancreatic MRI study including pre- and post-gadolinium T1-weighted sequences, T2-weighted sequences, and magnetic resonance cholangiopancreatography was performed for the definition of the local extension of poorly defined solid masses and the differentiation of cystic neoplasms [22, 23, 25].

EUS with fine-needle aspiration (FNA) provides highresolution images and relevant information about cytology and tumor markers of solid and cystic lesions, but the high operator dependency is the limit of the exam [21, 22]. EUS presents a high accuracy for small solid tumors, and EUS-FNA may be useful in the differential diagnosis of pancreatic cystic masses [25-27]. Over recent years, we have employed EUS more frequently in the diagnostic work-up of PIs.

Surgical resection is considered the standard treatment for asymptomatic pancreatic solid neoplasms $[1,6]$. Conversely, the management of cystic PIs is generally conservative due to the benign nature of the majority of 
Table 2 Surgical and follow-up data of 20 cases of left-sided pancreatic incidentalomas treated with laparoscopic approach

\begin{tabular}{|c|c|c|c|c|c|}
\hline No. & Surgery & Operative time (min) & Postop stay & Complications & Follow-up \\
\hline 1 & LDP & 204 & 7 & - & $A D$ \\
\hline 2 & LDP & 215 & 10 & $P, P E$ & $A D$ \\
\hline 3 & LSPDP & 228 & 5 & - & $A D$ \\
\hline 4 & LDP & 220 & 12 & POPF & $A D$ \\
\hline 5 & LDP & 217 & 10 & $P, P E$ & $A D$ \\
\hline 6 & LDP & 210 & 5 & - & $\mathrm{D}$ \\
\hline 7 & LDP-CO & 235 & 8 & PPD & D \\
\hline 8 & LDP & 191 & 11 & POPF, PE & D \\
\hline 9 & LDP & 188 & 9 & PPD & $D$ \\
\hline 10 & LDPDP & 198 & 5 & - & $A D$ \\
\hline 11 & LDP & 162 & 6 & - & $A D$ \\
\hline 12 & LDP & 238 & 8 & PPD & PD \\
\hline 13 & LDP & 172 & 14 & POPF & $\mathrm{D}$ \\
\hline 14 & LDPDP & 197 & 5 & - & $A D$ \\
\hline 15 & LDP-CO & 212 & 7 & PPD & $A D$ \\
\hline 16 & LDPDP & 230 & 6 & - & $A D$ \\
\hline 17 & LDP & 203 & 22 & POPF, PE & $D$ \\
\hline 18 & LDP & 185 & 6 & - & $A D$ \\
\hline 19 & LDP & 195 & 7 & - & $A D$ \\
\hline 20 & LDP & 200 & 9 & PPD & $A D$ \\
\hline
\end{tabular}

LDP laparoscopic distal pancreatectomy, LSPDP laparoscopic spleen-preserving distal pancreatectomy, $C O$, converted to open, $P E$ pleural effusion, $P O P F$ postoperative pancreatic fistula, $P$ pneumonia, $P P D$ post-pancreatectomy diabetes, $A D$ absence of disease, $P D$ progression of disease, $D$ died

these lesions [2]. Serous cystic neoplasm (SNC) is considered a benign lesion, and surgery should be considered only in case of large tumors (size $>4 \mathrm{~cm}$ ) or when preoperative exams are not conclusive [28]. Mucinous cystic neoplasms (MCN) and IPMN are frequently incidental [25]. Surgical resection is mandatory for $\mathrm{MCN}$ and main duct-type IPMN [29]. Branch duct-type IPMN should be considered for surgery only in case of a lesion greater than $3 \mathrm{~cm}$ associated with main duct dilatation $>10 \mathrm{~mm}$ or an enhanced solid component [29].

A relevant proportion of PIs are malignant or premalignant lesions [6, 7]. Malignancies detected incidentally are diagnosed in earlier stages, and long-term survivals seem to be more favorable than symptomatic lesions [7]. In our series, in six cases the histologic diagnosis of left-sided PI was DAC, and in two patients, it was ACC. Despite a complete preoperative diagnostic work-up, the final pathological staging demonstrated a relevant proportion of tumors with infiltration of surrounding tissues or microscopic lymph node involvement: four patients were classified as stage II A and three patients as stage II B. Consequently, when a pancreatic mass is detected, surgical treatment should be always considered [20].

The distal pancreatectomy consists in the resection of body and tail of the pancreas including the spleen and regional lymph nodes: it is considered the standard treatment of left-sided malignancies. Minimally invasive surgery was introduced for DAC staging, and subsequently, it was employed for pancreatic resections $[8,15]$. LDP is currently considered an effective treatment for benign and low-grade malignant lesions of the distal pancreas, but it is still debating if this technique is an appropriate treatment for DAC [8-11]. In the series presented in the literature, LDP was performed for non-malignant lesions in the majority of cases, but there are not clinical trials comparing longterm survivals of patients with DAC treated with laparoscopic or open approach [11]. In the meta-analysis of the literature, the tumor free margin rate and number of lymph nodes dissected are comparable in both techniques, but no definitive conclusions can be drawn about outcomes of laparoscopic resection for malignancy [9, 10]. However, LDP presents lower blood loss, shorter time to oral intake, and reduced length of hospital stay as compared to open surgery, while the rate of postoperative pancreatic fistulas is similar for the two surgical techniques [8-10].

We consider the presence of lymph node involvement and infiltration of surrounding tissues at preoperative work-up as contraindications to laparoscopic approach. In our series, conversion to open surgery 

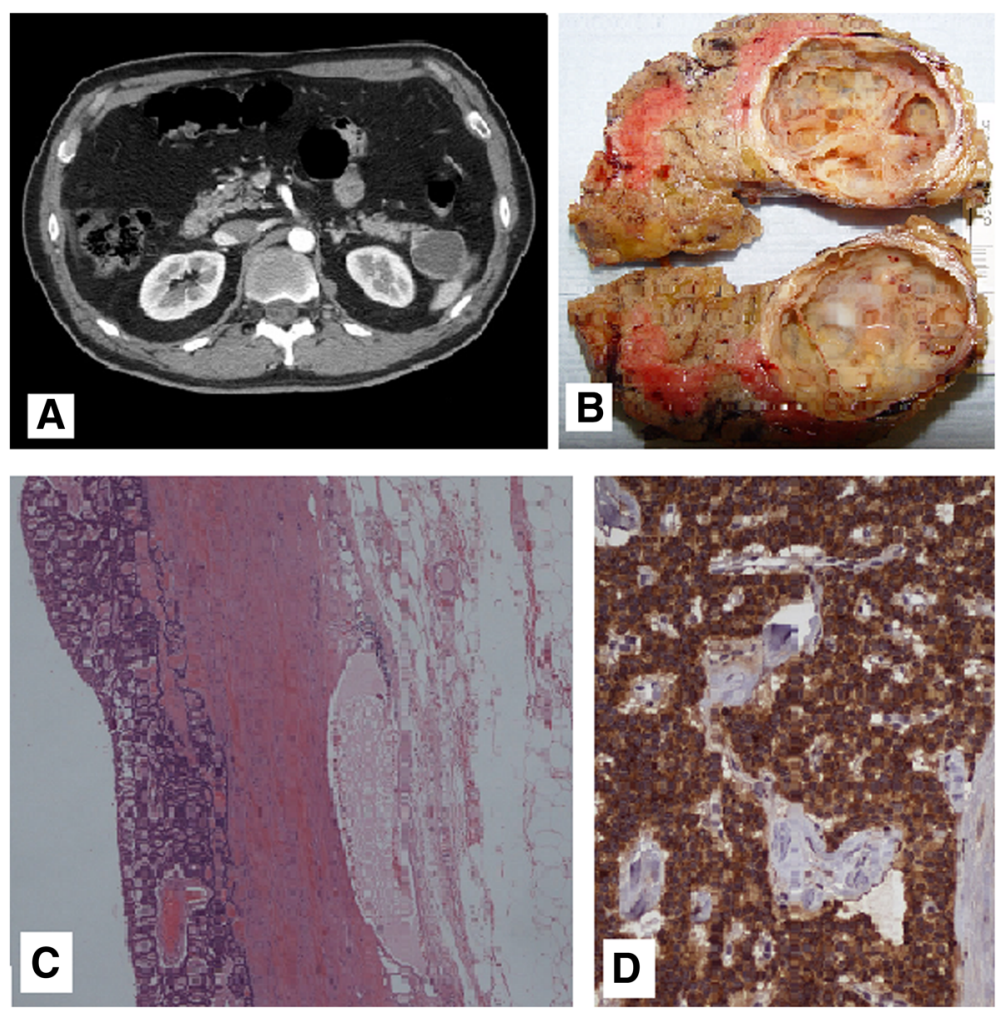

Fig. 1 Case 16. a CT scan demonstrating a $3 \times 4 \mathrm{~cm}$ pancreatic tail cystic lesion. b Surgical specimen of spleen-preserving distal pancreatectomy with a cystic lesion of the tail. A well-demarcated, solitary, and cystic mass of the pancreatic tail is a rare macroscopic presentation of a neuroendocrine tumor. c The characteristic trabecular and gyriform pattern of a pancreatic neuroendocrine tumor with relatively uniform cells (hematoxylin-eosin; magnification $\times 40$ ). $\mathbf{d}$ The immunohistochemical staining shows strong and diffuse expression of chromogranin $A($ magnification $\times 200$ )

was determined by the intraoperative detection of considerable-size masses causing the failure to progress. Laparoscopic spleen-preserving distal pancreatectomy was performed in two patients for small benign lesions localized in the tail. In patients with diagnosis of DAC, the number of lymph nodes removed was adequate for a correct oncologic resection.

The absence of postoperative deaths could be related to the epidemiologic features of our cohort: the series was composed by relatively young patients without considerable comorbidities.

In our series, the postoperative fistula rate was slightly high, but it was still in the range reported in the literature $[10,30]$. No grade $C$ fistula was observed, and in two cases, the pancreatic leak was only biochemical without any clinical relevance. Our high POPF rate may be attributed to lack of oversewing of the pancreatic stump, but any conclusions about the correlation
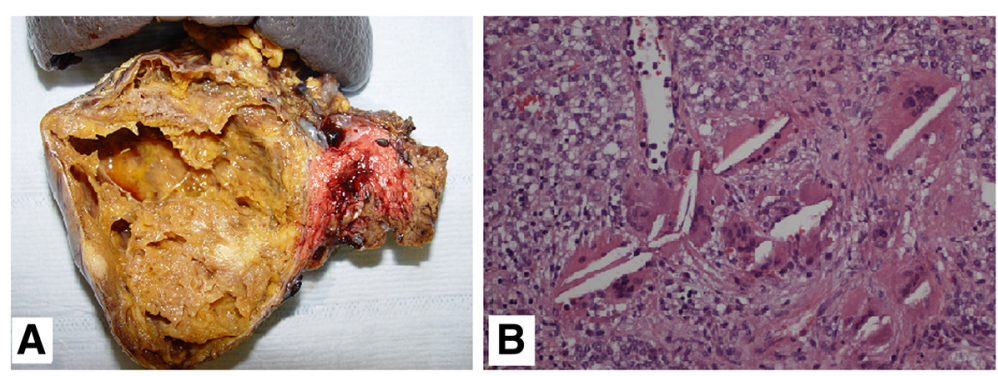

Fig. 2 Case 2. a Surgical specimen of distal pancreatectomy containing a 9.5-cm-large encapsulated pancreatic tail mass with areas of cystic degeneration. The histological diagnosis was solid pseudopapillary neoplasm. $\mathbf{b}$ The microscopic pattern of the neoplasm is solid and pseudopapillary with poorly cohesive monomorphic cells, admixed with thin-walled blood vessels. At the center of the image, there are characteristic cholesterol crystals, surrounded by foreign-body giant cells (hematoxylin-eosin; magnification $\times 200$ ) 

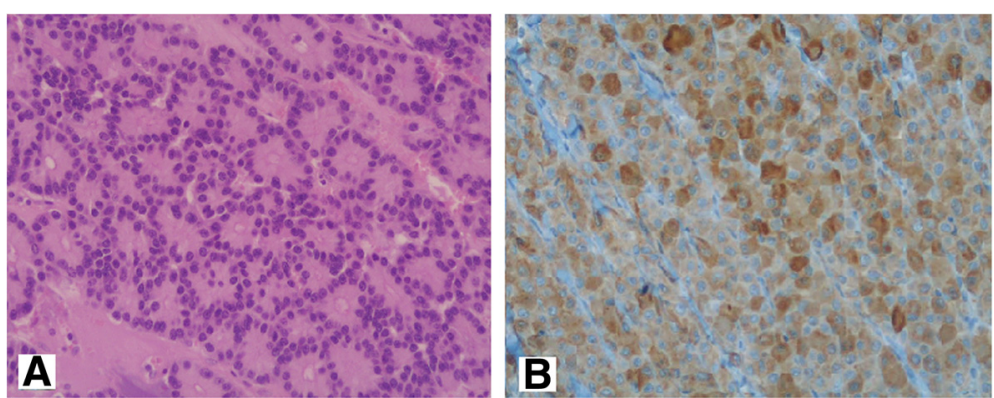

Fig. 3 Case 6. a Histological picture of an acinar cell carcinoma: the neoplasm composed by cells arranged in small acinar units (hematoxylin-eosin; magnification $\times 200$ ). $\mathbf{b}$ The immunohistochemical staining proves the expression of pancreatic exocrine enzymes (trypsin; magnification $\times 200$ )

between the type of surgical closure of the remnant gland and POPF cannot be drawn due to the limited number of the series.

Pleural effusion, a rare complication [8], was observed in four patients. In two cases, pleural effusion was associated with grade B pancreatic fistulas; in two cases was secondary to pneumonia, and it was self-limiting. Pleural and pulmonary complications are considered infrequent complications in pancreatic surgery, but a large observational study shows how LDP is associated with a pleuro-pulmonary morbidity rate of $26 \%$ [11].

The incidence of PPD was consistent with the data of the literature [31].

\section{Conclusions}

In conclusion, circumscribed incidentalomas of the distal pancreas in patients with minor comorbidities could be safely treated with laparoscopic approach. Left-sided PIs are frequently uncommon pancreatic neoplasms, but in a significant share of patients, DAC in early stages are found. In case of large-sized tumors or lymph node involvement, open surgery should be considered. Only clinical trials will confirm whether laparoscopic surgery is an effective treatment for malignant lesions [11].

\section{Abbreviations}

ACC, acinar cell carcinoma; ASA, American society of anesthesiologists; BMI, body mass index; $C$, computed tomography; DAC, ductal adenocarcinoma; $E D$, emergency department; EUS, endoscopic ultrasound; FNA, fine-needle aspiration; IPMN, intraductal papillary mucinous neoplasm; LDP, laparoscopic distal pancreatectomy; MCN, mucinous cystic neoplasm; MRI, magnetic resonance imaging; Pls, pancreatic incidentalomas; POPF, postoperative pancreatic fistulas; PPD, post-pancreatectomy diabetes; SCN, serous cystic neoplasm; US, ultrasonography

\section{Acknowledgements}

We thank Dr. Gerardo Cioffi, a native speaker, for reviewing the English language.

\section{Funding}

No source of funding has a role in the study's design, conduct, and reporting.

\section{Authors' contributions}

MC carried out the study, drafted the manuscript, and revised it. MG collected the information on the patients and wrote the contents of the methods and results of the manuscript. FT collected the information on the patients and revised the contents of the methods and results of the manuscript. LF helped in drafting the manuscript and revised the contents of the discussion of the manuscript. AG and FG collected the information on the patients and revised the contents of discussion of the manuscript. AM checked the histopathology and wrote the contents of histopatologic legends of the manuscript. MDS and UC carried out the concept and the design of the study and revised the manuscript. All authors read and approved the final manuscript.

\section{Competing interest}

The authors declare that they have no competing interests.

\section{Consent for publication}

Written informed consent was obtained from the patients of this study for the publication of personal data and images.

\section{Ethics approval and consent to participate}

The clinical study was approved by the Comitato Etico Interaziendale delle Province di Lecco, Como e Sondrio (no. 0018832/16U), and written informed consent to participate was obtained from the patients.

\section{Data sharing statement}

We provide as attachments the database containing all the information collected to perform the study and all documents related to it (ethics committee approval, informed consent, consent for the processing of personal data, study protocol, and synopses).

\section{Author details}

${ }^{1}$ Department of Surgery, Ospedale Alessandro Manzoni, Lecco, Italy. ${ }^{2}$ Department of Surgery, University of Milan-Bicocca, Istituti Clinici Zucchi, Via Zucchi, Monza, MB, Italy. ${ }^{3}$ Department of Pathology, Ospedale Alessandro Manzoni, Lecco, Italy. ${ }^{4}$ Department of Surgery, Istituto Clinico Humanitas Mater Domini, Castellanza, VA, Italy. ${ }^{5}$ Department of Surgery, University of Milan, Milan, Italy.

Received: 6 April 2016 Accepted: 13 July 2016

Published online: 04 August 2016

\section{References}

1. Zarate X, Williams N, Herrera MF. Pancreatic incidentalomas. Best Pract Res Clinical Endocrinol Metab. 2012;26:97-103.

2. Berland LL, Silverman SG, Gore RM, et al. Managing incidental findings on abdominal $\mathrm{CT}$ : white paper of the ACR incidental findings committee. J Am Coll Radiol. 2010;7:754-73.

3. Fitzgerald TL, Smith AJ, Ryan M, et al. Surgical treatment of incidentally identified pancreatic masses. Can J Surg. 2003;46:413-8.

4. Winter JM, Cameron JL, Lillemoe KD, et al. Periampullary and pancreatic incidentaloma. A single institution's experience with an increasingly common diagnosis. Ann Surg. 2006;243:673-80.

5. Bruzoni M, Johnston E, Sasson AR. Pancreatic incidentalomas: clinical and pathologic spectrum. Am J Surg. 2008;195:329-32.

6. Sachs T, Pratt WB, Callery MP, et al. The incidental asymptomatic pancreatic lesion: nuisance or threat? J Gastrointest Surg. 2009;13:405-15. 
7. Lahat $\mathrm{G}$, Ben Haim M, Nachmany I, et al. Pancreatic incidentalomas: high rate of potentially malignant tumors. J Am Coll Surg. 2009;209:313-9.

8. Borja-Cacho D, Al-Refaie WB, Vickers SM, et al. Laparoscopic distal pancreatectomy. J Am Coll Surg. 2009;209:758-65.

9. Jin T, Altaf $\mathrm{K}$, Xiong JJ, et al. A systematic review and meta-analysis of studies comparing laparoscopic and open distal pancreatectomy. HPB. 2012;14:711-24.

10. Venkat R, Edil BH, Schulick RD, et al. Laparoscopic distal pancreatectomy is associated with significantly less overall morbidity compared to the open technique: a systematic review and meta-analysis. Ann Surg. 2012;255: 1048-59.

11. Sulpice L, Farges O, Goutte N, et al. Laparoscopic distal pancreatectomy for pancreatic ductal adenocarcinoma: time for a randomized controlled trial? Results of an all-inclusive national observational study. Ann Surg. 2015;262:868-74.

12. Balzano G, Zerbi A, Capretti G, et al. Effect of hospital volume on outcome of pancreaticoduodenectomy in Italy. Br J Surg. 2008:95:357-62.

13. Bassi C, Dervenis $C$, Butturini $G$, et al. Postoperative pancreatic fistula: an international study group (ISGPF) group. Surgery. 2005;138:8-13.

14. American Diabetes Association. Diagnosis and classification of diabetes mellitus. Diabetes Care. 2012;35:564-71.

15. Patterson EJ, Gagner M, Salky B, et al. Laparoscopic pancreatic resection: single-institution experience of 19 patients. J Am Coll Surg. 2001;193:281-7.

16. Sener SF, Fremgen A, Menk HR, et al. Pancreatic cancer: a report of treatment and survival trends for 100,313 patients diagnosed from 19851995, using the National Cancer Database. J Am Coll Surg. 1999;189:1-7.

17. Crippa S, Fernandez-Del Castillo C, Salvia R, et al. Mucin-producing neoplasms of the pancreas: an analysis of distinguishing clinical and epidemiologic characteristics. Clin Gastroenterol Hepatol. 2010;8:213-9.

18. Zerbi A, Falconi M, Rindi G, et al. Clinicopathological features of pancreatic endocrine tumors: a prospective multicenter study in Italy of 297 sporadic cases. Am J Gastroenterol. 2010;105:1421-9.

19. De la Fuente SG, Ceppa EP, Reddy SK, et al. Incidence of benign disease in patients that underwent resection for presumed pancreatic cancer diagnosed by endoscopic ultrasonography (EUS) and fine-needle aspiration (FNA). J Gastrointest Surg. 2010;14:1139-42.

20. Sheehan M, Latona C, Araha G, et al. The increasing problem of unusual pancreatic tumors. Arch Surg. 2000;135:644-8.

21. Clarke DL, Thomson SR, Madiba TE, et al. Preoperative imaging of pancreatic cancer: a management-oriented approach. J Am Coll Surg. 2003;196:119-29.

22. Low G, Panu A, Millo N, et al. Multimodality imaging of neoplastic and nonneoplastic solid lesions of the pancreas. Radiographics. 2011;31:993-1015.

23. Buerke B, Domagk D, Heindel W, et al. Diagnostic and radiological management of cystic pancreatic lesions: important features for radiologists. Clin Radiol. 2012:67:727-37.

24. Kocher KE, Meurer WJ, Fazel R, et al. National trends in use of computed tomography in the emergency department. Ann Emerg Med. 2011;58:452-62.

25. Italian Association of Hospital Gastroenterologists and Endoscopists; Italian Association for the Study of the Pancreas, Buscarini E, Pezzilli R, Cannizzaro R, et al. Italian consensus guidelines for the diagnostic work-up and follow-up of cystic pancreatic neoplasms. Dig Liver Dis. 2014:46:479-93.

26. Petrone MC, Arcidiacono PG. Role of endoscopic ultrasound in the diagnosis of cystic tumours of the pancreas. Dig Liver Dis. 2008:40:847-53.

27. Khalid A, Brugge W. ACG practice guidelines for the diagnosis and management of neoplastic pancreatic cysts. Am J Gastroenetrol. 2007; 102:2339-49.

28. Sakorafas GH, Smyrniotis V, Reid-Lombardo KM, et al. Primary pancreatic cystic neoplasms revisited. Part I: serous cystic neoplasms. Surg Oncol. 2011;20:e84-92.

29. Tanaka M, Fernandez-del Castillo C, Adsay V, et al. International consensus guidelines 2012 for the management of IPMN and MCN of the pancreas. Pancreatology. 2012;12:183-97.

30. Natan H, Cameron JL, Goodwin CR, et al. Risk factors for pancreatic leak after distal pancreatectomy. Ann Surg. 2009;250:277-81.

31. Shirakawa S, Matsumoto I, Toyama H, et al. Pancreatic volumetric assessment as a predictor of new-onset diabetes following distal pancreatectomy. J Gastrointest Surg. 2012;16:2212-9.

\section{Submit your next manuscript to BioMed Central and we will help you at every step:}

- We accept pre-submission inquiries

- Our selector tool helps you to find the most relevant journal

- We provide round the clock customer support

- Convenient online submission

- Thorough peer review

- Inclusion in PubMed and all major indexing services

- Maximum visibility for your research

Submit your manuscript at www.biomedcentral.com/submit
Biomed Central 